\title{
3 Research Square

\section{R248Q mutation of p53 alters molecular trafficking and targeted drug responses in high-grade serous ovarian carcinoma}

\section{Kai-Yun Tsai}

National Tsing Hua University

Zih-Yin Lai

National Tsing Hua University

Shing-Jyh Chang

Mackay Memorial Hospital Hsinchu

Yung-Jen Chuang ( $\nabla$ yjchuang@life.nthu.edu.tw )

National Tsing Hua University https://orcid.org/0000-0002-6022-0107

Research article

Keywords: p53; epidermal growth factor receptor (EGFR); AKT; mouse double minute 2 homolog (MDM2); combination therapy

Posted Date: September 30th, 2019

DOI: https://doi.org/10.21203/rs.2.15415/v1

License: (9) This work is licensed under a Creative Commons Attribution 4.0 International License. Read Full License 


\section{Abstract}

Background p53 mutations are detected in up to $96 \%$ of high-grade serous ovarian carcinoma (HGSOC). Moreover, mutant p53 may cause oncogenic gain-of-function phenotypes in sustained activation of epidermal growth factor receptor (EGFR) signaling. In this study, we investigated whether p53 mutation could affect EGFR-related signaling and the corresponding therapeutic strategies in HGSOC.Methods For this study, we selected the p53 R248Q mutant (p53 R248Q), which has the highest mutation frequency in ovarian cancer. Since we have previously demonstrated that the combined inhibition of EGFR and MDM2p53 pathways by gefitinib and JNJ-26854165 exerts a strong synergistic lethal effect on p53-mutated HGSOC cells, the gefitinib and JNJ-26854165 responses in different p53 status cells were evaluated by cell viability and target protein expression assays. Results We showed that the phosphorylation of AKT, a critical molecule of EGFR downstream signaling, increased significantly when p53 R248Q was transiently overexpressed. Immunocytochemistry analysis further showed that upon p53 R248Q overexpression, several AKT-dependent mediators translocate in unique patterns within the cell. Subsequent analysis revealed that under the combined inhibition of gefitinib and JNJ-26854165, the cytonuclear trafficking of EGFR and MDM2 is disrupted. Moreover, when we further compared gefitinib and JNJ-26854165 responses in different p53 status cells, we found that the sensitivity to the single- or combined-inhibition treatments is also altered in p53 R248Q -overexpressing cells.Conclusions Our findings suggest that the R248Q mutation of p53 causes significant changes in signaling transduction, molecular trafficking and drug responses, which help to advance our understanding of p53 and to improve therapeutic strategies for HGSOC.

\section{Background}

Epithelial ovarian cancer is the most prevalent ( 90\%) type of ovarian carcinoma [1] and could be further divided into subtypes based on histopathology, gene alterations, and prognosis [2]. Among the five main subtypes, high-grade serous ovarian carcinoma (HGSOC) accounts for the highest proportion ( 70\%) [3]. HGSOC is known to have a high risk of recurrence and platinum-based chemotherapy drug resistance due to genetic changes in tumor suppressor p53 encoded by TP53 [4]. As one would expect, ovarian cancer has the highest rate of somatic TP53 mutation among all cancers [5]. In fact, TP53 is mutated in up to $96 \%$ of HGSOC, with arginine 248 (R248) being the most common mutated amino acid [6].

p53 also acts as a transcription factor, with most mutations being located in its DNA-binding domain (DBD) $[7,8]$. In addition, it has been demonstrated that mutant p53 not only loses its activities but also displays oncogenic gain-of-function, such as accelerating tumor progression and acquiring drug resistance [9]. Since mutant p53 is known to amplify receptor tyrosine kinase (RTK) signaling [10-15], the association between $\mathrm{p} 53^{\mathrm{R} 248 \mathrm{Q}}$ and RTK-regulated signaling in HGSOC could be important for cancer progression. Among the RTK downstream signaling pathways, several studies have revealed that p53 ${ }^{\mathrm{R} 248 \mathrm{Q}}$ promotes cell migration and proliferation through AKT signaling $[16,17]$. However, how the R248Q mutation of p53 affects AKT-dependent carcinogenesis has not been elucidated. 
Excessive activation of AKT signaling can mediate a variety of cellular processes, including cell cycle dysregulation, proliferation, and drug resistance, all of which are considered hallmarks of cancer [18]. In addition, it has been elucidated that activated AKT translocates to various organelles, where AKT phosphorylates substrates or interacts with other factors to regulate a complex network of processes [19]. For instance, AKT phosphorylates MDM2 on Serine 166 and Serine 186 to initiate MDM2's nuclear translocation. MDM2 then acts to promote $\mathrm{p} 53$ ubiquitination for degradation and reduce p53 transcriptional activities [20]. Similarly, phosphorylation of EGFR's Serine 229 by AKT also promotes nuclear translocation of EGFR [21], which acts as a co-transcription factor of multiple genes regulating cell proliferation and tumor angiogenesis, resulting in enhanced drug resistance and poor patient outcomes [22]. It should be noted that whether the R248Q mutation of p53 affects AKT-dependent molecular trafficking has not been determined.

The taxane- and platinum-based chemoresistance in ovarian cancer is known to be associated with its high mortality rates [23]. Moreover, numerous studies have indicated that up to $60 \%$ of epithelial ovarian cancer displays EGFR overexpression. However, treatment of ovarian cancers with the anti-EGFR agent gefitinib alone generates a limited response [24]. When gefitinib is used together with platinumbased chemotherapy drugs, the combination therapy would increase the overall response rate in patients with relapsed ovarian cancer [25]. However, such combination therapy has exhibited limited efficacy in other clinical trials [26]. Such finding suggests a need to modify the treatment scheme.

Previous studies have shown that up to $80 \%$ of ovarian serous borderline tumors exhibit MDM2 overexpression [27], while the expression of MDM2 is notably low in benign ovarian tumors or normal ovaries [28]. Biomarker analysis has found that the co-expression of p53 and MDM2 is associated with poor outcomes in epithelial ovarian cancer patients $(P<0.05)$ [29]. Furthermore, our previous study demonstrated that the combined inhibition of EGFR and MDM2-p53 pathways in HGSOC could generate a strong synergistic anti-cancer effect [30]. These findings led us to explore whether the R248Q mutation of p53 would affect the drug efficacy and the underlying mechanisms by which EGFR and MDM2-p53 interact to produce a synergistic effect.

In this study, we first investigated how the R248Q mutation of p53 alters the characteristics of HGSOC. We then explored how the R248Q mutation of p53 regulates the drug responses and synergistic effect under the combined inhibition of EGFR and MDM2.

\section{Methods}

\subsection{Cell culture}

The serous ovarian cancer cell line used in this study was OVCAR3 ( $\mathrm{p} 53^{\mathrm{R} 248 \mathrm{Q}}$ mutation). The TP53 status was annotated according to the International Agency for Research on Cancer (IARC) TP53 database (http://p53.iarc.fr/). The cells were cultured at $37^{\circ} \mathrm{C}$ with $5 \% \mathrm{CO}_{2}$ in RPMI-1640 medium (Gibco) supplemented with $10 \%$ fetal bovine serum. 


\subsection{Drugs and reagents}

Gefitinib (LC Laboratories) and JNJ-26854165 (AdooQ BioScience) were dissolved in DMSO and stored at $-20^{\circ} \mathrm{C}$. EGF (Sigma-Aldrich) was dissolved in $0.2-\mu \mathrm{m}$-filtered $10 \mathrm{mM}$ acetic acid and stored at $-20^{\circ} \mathrm{C}$.

\subsection{Gene knockdown and overexpression}

siRNAs for $\mathrm{p} 53$ and control siRNA were synthesized by Genepharma. Cells were transfected with siRNA at a final concentration of $25 \mathrm{nM}$ using lipofectamine (Invitrogen) following the manufacturer's protocols. The $\mathrm{p} 53^{\mathrm{R} 248 \mathrm{Q}}$ targeting plasmid was constructed in house. Cells were transfected with plasmid at a final concentration of $1 \mu \mathrm{g} / \mathrm{mL}$ using jetPRIME (Polyplus) following the manufacturer's protocols.

\subsection{Western blot analysis}

Cell lysates were extracted using RIPA lysis buffer (Roche). The products were then quantitated with a BCA protein assay kit (Pierce). Proteins were separated using $8 \%$ or $12 \%$ SDS-PAGE and then transferred to PVDF membranes (Millipore). Transferred membranes were immersed in 3\% bovine serum albumin (BSA) dissolved in $1 \mathrm{X}$ TBS with $0.05 \%$ Tween-20 for 1 hour at room temperature. The PVDF membranes were incubated with different primary antibodies diluted in blocking solution overnight at $4^{\circ} \mathrm{C}$ and then incubated with secondary antibodies (GeneTex) diluted in 5\% nonfat milk/TBST at room temperature for 2 hours before signal detection. Finally, the signal was enhanced by Amersham ECL Prime Western Blotting Detection Reagent (GE Healthcare). The anti-pEGFR, pAKT, AKT, pMAPK, MAPK, and p21 antibodies were obtained from Cell Signaling Technology. The anti-EGFR, p53 and GAPDH antibodies were obtained from GeneTex.

\subsection{Immunocytochemistry (ICC)}

OVCAR3 cells were seeded on sterile glass coverslips prior to the experiments. Cells were fixed with $4 \%$ paraformaldehyde/PBS for 10 minutes and permeabilized with $0.1 \%$ Triton X-100/PBS for another 10 minutes. Then, the cells were blocked with $3 \%$ BSA/PBS for 30 minutes. The primary antibodies were then added directly into blocking buffer and incubated overnight at $4^{\circ} \mathrm{C}$. Afterward, the secondary antibodies, which were anti-rabbit IgG-DyLight488 (Jackson) and anti-mouse IgG-DyLight649 (Jackson), were added and incubated for 2 hours at $37^{\circ} \mathrm{C}$. The nucleus and actin cytoskeleton were stained with Hoechst 33342 (Invitrogen) and phalloidin (Invitrogen), respectively. The images of stained cells were captured by confocal microscopy (LSM510 Meta, Zeiss). The anti-EGFR (green signal), AKT, and FOXO3a antibodies 
were obtained from Cell Signaling Technology. The anti-EGFR (purple signal) and MDM2 antibodies were obtained from GeneTex. The anti-mutant p53 antibody was obtained from Merck.

\subsection{Cell viability assay}

OVCAR3 cells were seeded in $96-$-well plates at a density of $5 \times 10^{3}$ cells/well. Cells were transfected with empty plasmid only $(1 \mu \mathrm{g} / \mathrm{mL})$ or with the ${ }^{2} 53^{\mathrm{R} 248 \mathrm{Q}}$ construct $(1 \mu \mathrm{g} / \mathrm{mL})$ for 18 hours and then treated with drugs of different concentrations for 48 hours. Following the manufacturer's protocols, $10 \mu \mathrm{L}$ Cell Counting Kit-8 (Dojindo) cell proliferation reagent was added to 96 -well plates and incubated at $37^{\circ} \mathrm{C}$ for 1 to 2 hours. The optical densities to reflect cell viability were determined by absorbance wavelength at $450 \mathrm{~nm}$.

\subsection{Analysis of combination index}

The combination index $(\mathrm{Cl})$ values were calculated by CompuSyn calculation software as described by Chou and Talalay [31]. First, the cell viability assay data were converted into the fraction affected ( $\mathrm{Fa})$ under specified drug treatment conditions. Next, the Fa values of single agents or drug combinations were uploaded into CompuSyn and generated the $\mathrm{Cl}$ values, the $\mathrm{Fa}-\mathrm{Cl}$ plots and the isobolograms as instructed by the user manual.

\subsection{Statistics}

Data were processed by GraphPad Prism 6 and expressed as the means \pm SEM (standard error of the mean). Statistical significance was determined by $p$ values $<0.05$ using Student's t-test.

\section{Results}

\subsection{R248Q mutation of $p 53$ amplified p-AKT signaling in ovarian cancer cells}

We first investigated whether $\mathrm{p} 53^{\mathrm{R} 248 \mathrm{Q}}$ affected EGFR (a member of the RTK family) and its downstream signaling in OVCAR3 cells (HGSOC cell line with $p 53^{R 2480}$ ). Since EGFR is aberrantly overexpressed and activated in ovarian cancer [32,33], we stimulated pre-starved cancer cells with epidermal growth factor (EGF) to activate the EGFR signaling. Both activated PI3K/AKT and MAPK/ERK pathways under EGF stimulation have been implicated in various cancers. Therefore, we examined these EGFR downstream markers under the condition of p53 siRNA knockdown. We found that p-AKT and pMAPK decreased in OVCAR3 cells after $p 53^{R 248 Q}$ knockdown (Supplementary Fig. S1A). Using an overexpression approach, we found that p-AKT was significantly upregulated after augmented expression 
of $p 53^{\mathrm{R} 248 \mathrm{Q}}$ in OVCAR3 cells (Supplementary Fig. S1B). Taken together, these data indicated that the R248Q mutation of p53 amplified p-AKT signaling in OVCAR3 cells.

\section{2. $\mathrm{p} 53^{\mathrm{R} 248 \mathrm{Q}}$ overexpression and EGF stimulation resulted in similar cytonuclear trafficking of $A K T$, EGFR, MDM2, and FOXO3a}

AKT signaling is known to mediate intracellular trafficking of various receptors and regulatory proteins. We thus investigated how the R248Q mutation of p53 alters the intracellular molecular trafficking of EGFR, MDM2, and FOXO3a in association with AKT (Fig. 1).

We found that, after either EGF stimulation or $\mathrm{p} 53^{\mathrm{R} 248 \mathrm{Q}}$ overexpression, most of the AKT translocated into the nucleus completely. However, when $\mathrm{p} 53^{\mathrm{R} 248 \mathrm{Q}}$ overexpression was combined with EGF stimulation, some AKT remained in the cytoplasm (Fig. 1A). Consistent with past studies, our data implied that AKT enters the nucleus in response to growth factors to exert regulatory activities [34-36]; thus, we continued to investigate how AKT translocation correlates with the intracellular trafficking of EGFR and MDM2.

Similar but different from the aforementioned findings, after EGF stimulation or $\mathrm{p} 53^{\mathrm{R} 248 \mathrm{Q}}$ overexpression, both EGFR and MDM2 appeared to converge and translocate to the periphery of the cell nucleus (Fig. 1B and 1C). In comparison to the control group, we also observed some EGFR still localized on the cell membrane, while most of the MDM2 translocated to the periphery of the cell nucleus.

Interestingly, when $\mathrm{p} 53^{\mathrm{R} 248 \mathrm{Q}}$ overexpression was combined with EGF stimulation, the EGFR located on the cell membrane and the MDM2 clustering around the nucleus were not detected, and most of the MDM2 translocated into the cell nucleus.

On the other hand, it has been reported that FOXO3a, a member of the forkhead box 0 (FoxO) transcription factor families and a tumor suppressor, accelerates its nuclear export when it is phosphorylated by nuclear AKT [37]. Therefore, we speculated that this tumorigenic marker is affected by $\mathrm{p} 53^{\mathrm{R} 248 \mathrm{Q}}$. Based on our data, we found that FOXO3a, which mostly localized in the nucleus in the control group, distributed uniformly in the cytoplasm under the following conditions: after EGF stimulation, with $\mathrm{p} 53^{\mathrm{R} 248 \mathrm{Q}}$ overexpression, and $\mathrm{p} 53^{\mathrm{R} 248 \mathrm{Q}}$ overexpression combined with EGF stimulation (Fig. 1D).

These findings suggest that the R248Q mutation of p53 promotes tumorigenesis-related AKT signaling by affecting molecular intracellular trafficking.

\subsection{Combined blockade by gefitinib and JNJ attenuated EGFR and MDM2 cytonuclear trafficking}

The comparable translocation patterns of EGFR and MDM2 implied that the molecular cytonuclear trafficking may relate to the synergistic effect of combined EGFR and MDM2 inhibition that has been 
reported in our previous study [30]. In this present study, we again used gefitinib (EGFR tyrosine kinase inhibitor) and JNJ-26854165 (referred to as JNJ; MDM2 E3 ubiquitin ligase domain inhibitor) to determine whether such combined inhibition would alter the intracellular localization patterns of EGFR and MDM2.

By immunocytochemistry staining analysis, we first reconfirmed that both EGFR and MDM2 would translocate from the cytoplasm to the nucleus in accordance with each other over time after EGF stimulation (Supplementary Fig. S2). Next, we found that such nuclear convergence of EGFR was attenuated under treatment with gefitinib, JNJ, or both. Interestingly, the nuclear convergence of MDM2 was only disrupted under the combination treatment of $\mathrm{JNJ}$ and gefitinib (Fig. 2A and 2B). These results indicate that the combined inhibition of gefitinib and JNJ could prevent the converging nuclear translocation of EGFR and MDM2, which might regulate further signaling and oncogenic activity.

\subsection{R248Q mutation of p53 increased the sensitivity of EGFR and MDM2 inhibitors}

To further explore the impact of $\mathrm{p} 53^{\mathrm{R} 248 \mathrm{Q}}$ on the cellular response to combination therapy for HGSOC, we evaluated the anti-cancer efficacy of gefitinib and JNJ in OVCAR3 cells with different p53 statuses.

As shown in Supplementary Table 1, the combined inhibition of EGFR and MDM2 significantly reduced the $\mathrm{IC}_{50}$ values compared to the single agent treatment. Interestingly, the $\mathrm{IC}_{50}$ values of $\mathrm{JNJ}$ and gefitinib alone reduced to $66 \%$ (from 21.83 to $14.35 \mu \mathrm{M}$ ) and $78 \%$ (from 33.74 to $26.47 \mu \mathrm{M}$ ) when the cells were

overexpressing $\mathrm{p} 53^{\mathrm{R} 248 \mathrm{Q}}$. On the other hand, the combined inhibition resulted in a slight reduction (from 8.57 to $8.18 \mu \mathrm{M})$ of $\mathrm{IC}_{50}$.

For a more intuitive comparison, we summarized the data as Fig. 3 to highlight the significant reduction in effective drug concentration under combined inhibition. A synergistic effect was observed whether cells were pre-transfected with the $\mathrm{p} 53^{\mathrm{R} 248 \mathrm{Q}}$ plasmid or not (Fig. 3A, 3B). Interestingly, gefitinib and JNJ alone were more effective when OVCAR3 cells were pre-transfected with the $\mathrm{p} 53^{\mathrm{R} 248 \mathrm{Q}}$ plasmid (Fig. $3 \mathrm{C}$, 3D). However, the combined inhibition effect remained similar whether OVCAR3 cells were pre-transfected with the $\mathrm{p} 53^{\mathrm{R} 248 \mathrm{Q}}$ plasmid or not (Fig. 3E). Taken together, these results suggest that the R248Q mutation of p53 increases the sensitivity of OVCAR3 cells to EGFR and MDM2 inhibition to various degrees.

\subsection{R248Q mutation of p53 decreased the synergistic lethal effect of gefitinib and JNJ}

To better evaluate the synergistic effects exerted by combined inhibition in different p53 statuses, we adapted the well-known Chou and Talalay's combination index ( $\mathrm{Cl}$ ) method to perform in-depth analysis. It should be noted that a $\mathrm{Cl}$ value less than 1 is defined as drug synergism $[31,38]$. 
The $\mathrm{Cl}$ analysis revealed that in the absence of overexpression of $\mathrm{p} 53^{\mathrm{R} 248 \mathrm{Q}}$, the area below the $\mathrm{Cl}=1$ line was larger than that of the $\mathrm{p} 53^{\mathrm{R} 248 \mathrm{Q}}$ overexpression group (Fig. 4A and $4 \mathrm{~B}$ ). We also generated isobolograms to quantify the drug synergism at different effective doses (EDs). In comparison to the $\mathrm{p} 53^{\mathrm{R} 248 \mathrm{Q}}$ overexpression group, the symbols (colored as specified) were farther from the corresponding colored lines, and the $\mathrm{Cl}$ values were also lower in the absence of overexpression of $\mathrm{p} 53^{\mathrm{R} 248 \mathrm{Q}}$ (Supplementary Table 2, Fig. 4C, 4D). The $\mathrm{Cl}$ values and the synergism grading are summarized in Supplementary Table 2.

In OVCAR3 cells overexpressing $\mathrm{p} 53^{\mathrm{R} 248 \mathrm{Q}}$, the drug combination displayed slight synergism $(\mathrm{Cl}=0.87)$ at $\mathrm{ED}_{50}$, but changed to moderate $(\mathrm{Cl}=0.72)$ and enhanced synergism $(\mathrm{Cl}=0.67)$ at $\mathrm{ED}_{75}$ and $\mathrm{ED}_{90}$. Interestingly, OVCAR3 cells without $\mathrm{p} 53^{\mathrm{R} 248 \mathrm{Q}}$ overexpression showed enhanced synergism $(\mathrm{Cl}=0.65)$ at lower doses $\left(E_{50}\right)$. This finding implied that a higher dose of $J N J$ and gefitinib $\left(E D_{90}\right)$ was required to reach synergism in OVCAR3 cells overexpressing $\mathrm{p} 53^{\mathrm{R} 248 \mathrm{Q}}$. In contrast, lower dose of drug combination (i.e. $\mathrm{ED}_{50}$ ) exerted synergism in OVCAR3 cells without $\mathrm{p} 53^{\mathrm{R} 248 \mathrm{Q}}$ overexpression. Such data supports that overexpression of $\mathrm{p} 53^{\mathrm{R} 248 \mathrm{Q}}$ may attenuate the synergistic lethal effect of JNJ and gefitinib.

\subsection{MAPK and p21 regulated the effects of single and combined treatment of gefitinib and JNJ}

To provide more clues about the regulatory effect of $\mathrm{p} 53^{\mathrm{R} 248 \mathrm{Q}}$ on the observed differential sensitivity to JNJ and gefitinib, we analyzed the expression profiles of MAPK and p21 (a p53 target gene associated with cell cycle and apoptosis pathways) under different conditions of JNJ and gefitinib concentration and combination.

As expected, the enhanced expression of p53 was detected, which served to validate the overexpression of $\mathrm{p} 53^{\mathrm{R} 248 \mathrm{Q}}$ after transfection. Next, the results revealed that $\mathrm{p}-\mathrm{MAPK}$ was significantly reduced by gefitinib in a dose-dependent manner (Fig. 5A). Moreover, at a dose of $10 \mu \mathrm{M}$, gefitinib exerted a stronger MAPK-reduction effect in $\mathrm{p} 53^{\mathrm{R} 248 \mathrm{Q}}$-overexpressing cells compared to the cells without overexpression (Supplementary Fig. S3A). We also found that the expression level of p21 was significantly increased by $\mathrm{JNJ}$ in a dosage-dependent manner. Upon examination and comparison of the two DMSO control groups, we found that the expression level of p21 was lower in p53 ${ }^{\mathrm{R} 248 \mathrm{Q}}$-overexpressing cells (Fig. 5B). However, with increasing $\mathrm{JNJ}$ dose, $\mathrm{p} 21$ increased significantly (approximately 17 -fold) in p53 ${ }^{\mathrm{R} 248 \mathrm{Q}}$-overexpressing cells compared to the cells without overexpression (approximately 4-fold) (Supplementary Fig. S3B). Strikingly, the effects were reversed when JNJ was combined with gefitinib (Fig. 5C, Supplementary Fig. S3C, S3D).

These results imply that in the presence of $\mathrm{p} 53^{\mathrm{R} 248 \mathrm{Q}}$ overexpression, the augmented sensitivity to $\mathrm{JNJ}$ or gefitinib and the weakened synergistic effect of combined treatment might be associated with the alternating expression of MAPK and $\mathrm{p} 21$. 


\section{Discussion And Conclusions}

In this study, we provide new evidence to highlight the effects of the R248Q mutation of p53 on cell signaling regulation and the response to EGFR/MDM2 targeted therapies in HGSOC.

Mutant p53 is generally considered to accelerate carcinogenesis by carrying out a dominant-negative effect on wild-type p53 and by manifesting gain-of function activities [39]. In this study, our findings iterated that the phosphorylation of AKT would be amplified in OVCAR3 cells via $p 53^{R 248 Q}$ overexpression (Supplementary Fig. S1). For the effects of enhanced p-AKT signaling in HGSOC, we first speculated that the subcellular localization of AKT, as well as AKT-dependent molecular trafficking, may be altered under the influence of $p 53^{R 248 Q}$. This hypothesis is supported by the evidences that nuclear AKT induced by various growth factors and stimuli has been shown to promote tumorigenesis by controlling cell cycle progression, promoting cell survival and DNA repair, and counteracting apoptosis [19]. In this study, we have further examined the trafficking patterns of AKT, EGFR, MDM2, and FOXO3a upon EGF stimulation and $\mathrm{p} 53^{\mathrm{R} 248 \mathrm{Q}}$ overexpression (Fig. 1). Our data reveals that these molecules' trafficking patterns under $p 53^{\mathrm{R} 248 \mathrm{Q}}$ overexpression exert a comparable effect to that of EGF stimulation. Based on our findings, we propose a hypothetical model of molecular trafficking in response to EGF stimulation and $p 53^{R 248 Q}$ overexpression (Fig. 6A).

In this modeled scheme, the R248Q mutation of p53 could amplify the phosphorylation of AKT, similar to the stimulation of EGF. The resulting p-AKT would further phosphorylate EGFR and MDM2 to facilitate their converging nuclear translocation. Furthermore, nuclear AKT would further phosphorylate FOXO3a to dispel it from the nucleus. Surprisingly, the combined $\mathrm{p} 53^{\mathrm{R} 248 \mathrm{Q}}$ overexpression and EGF stimulation did not lead to augmentation of nuclear AKT. In contrast, such combined treatment could promote more EGFR and MDM2 to translocate toward or into the nucleus. To the best of our knowledge, such phenomena have not been reported previously. We speculated that the AKT phosphorylation induced by EGFR activation and $\mathrm{p} 53^{\mathrm{R} 248 \mathrm{Q}}$ results in antagonism, while the duel phosphorylation induced by nuclear AKT and EGF on EGFR leads to synergism. The difference in the translocation pattern of MDM2 may arise from the differential signaling of nuclear AKT and cytoplasmic AKT. The underlying mechanisms shall be further explored in future studies.

To view this study in a broader context, evidences accumulated over the past 20 years have helped to characterize the function of nuclear AKT, which phosphorylates various transcription factors, including members of the FOXO transcription factor families [40]. The phosphorylated FOXO3a would be expelled from the nucleus and weakened its transcriptional capacity. As a result, the expression level of its target gene, such as p21, was reduced [41](Fig. 5). Nuclear EGFR has been shown to act as a co-transcription 
factor alongside STAT3, E2F1 and STAT5 to initiate the transcription of several cell cycle regulatory genes, such as Cyclin D1, Aurora A, and Myc [22]. Nuclear MDM2 mainly diminishes cellular levels of p53 and inhibits its transcriptional activity $[20,42,43]$. Therefore, the nuclear localization of AKT, EGFR, and MDM2 and the exclusion of nuclear FOXO3a have been linked with enhanced drug resistance, poor prognosis, and unfavorable overall survival in various cancers $[22,44-47]$.

To date, the combination of paclitaxel- and platinum-based drugs is a standard first-line treatment for ovarian cancer. However, chemoresistance to these drugs may lead to an unfavorable 5-year survival rate in advanced patients [48]. To overcome drug resistance, we suggested a novel combination therapy strategy with EGFR and MDM2 inhibition in our previous study [30]. In the present study, we further identified that the disruption of cytonuclear trafficking by the dual inhibition of EGFR and MDM2 might contribute to the synergistic effect of gefitinib and JNJ (Fig. 2). In addition, we demonstrated that the R248Q mutation of p53 enhanced the efficacy of gefitinib and JNJ alone, but reduced the synergistic effect of their combination (Fig. 3 and 4). Our data further validated this observation by showing the differential effect of p-MAPK inhibition and the differential effect of p21 induction under different gefitinib and JNJ treatment conditions (Fig. 5). These findings suggested that the degree of synergism of gefitinib and JNJ depends not only on the cytonuclear trafficking of signaling mediators but also on the mutation of p53.

Based on our findings, the proposed model (Fig. 6B) underlines the mechanistic roles of p-MAPK and p21 under the treatment of gefitinib and JNJ, either in the absence or presence of $p 53^{R 248 Q}$ overexpression. It should be noted that MDM2 is known to ubiquitinate the pro-apoptotic transcription factor FOXO3a via activated MAPK signaling [49]. As expected, gefitinib inhibited its downstream molecule $\mathrm{p}-\mathrm{MAPK}$ in the absence of $\mathrm{p} 53^{\mathrm{R} 248 \mathrm{Q}}$ overexpression. The reduction of p-MAPK and upregulation of p21 was controlled by EGFR inhibition and the negative feedback of MDM2. On the other hand, with $p 53^{R 248 Q}$ overexpression, gefitinib may cooperate with $p 53^{R 248 Q}$ to reduce $p-M A P K$. Our model further explains how the reduction of $\mathrm{p}-\mathrm{MAPK}$ is primarily influenced by $\mathrm{p} 53^{\mathrm{R} 248 \mathrm{Q}}$ and secondarily influenced by MDM2. Hence, the relevant cell signaling would be disrupted by JNJ, while gefitinib in the presence of p53 ${ }^{\mathrm{R} 248 \mathrm{Q}}$ overexpression could not exert its expected effects on p-MAPK and p21 (Fig. 6B). This model has the potential to revise the therapeutic approach for cancer treatment, which suggests the use of gefitinib or $\mathrm{JNJ}$ alone if the R248Q mutation of p53 is detected in HGSOC patients; if the R248Q mutation of $\mathrm{p} 53$ is not detected, the combination of gefitinib and JNJ should be recommended. While additional work is needed to confirm these predictions, we foresee that further understanding of the relationship between p53 mutation and the altered regulatory mechanisms could enhance our knowledge of ovarian carcinogenesis and the implementation of precision cancer medicine. 


\section{Abbreviations}

Cl: combination index; $\mathrm{ED}$ : effective dose; $\mathrm{ED}_{50,75,90}: 50 \%, 75 \%, 90 \%$ effective dose; EGF: epidermal growth factor; EGFR: epidermal growth factor receptor; Fa: fraction of affected cells; FOXO3a: forkhead box 03; HGSOC: high-grade serous ovarian carcinoma; $\mathrm{IC}_{50}$ : half maximal inhibitory concentration; JNJ: JNJ-26854165, also known as Serdemetan; MAPK: mitogen-activated protein kinase, also known as ERK; MDM2: mouse double minute 2 homolog; p53 ${ }^{\text {2448: }}$ p53 R248Q mutant; $\mathrm{p}-\mathrm{AKT}$ : phosphorylated AKT; PI3K: phosphoinositide 3-kinase; p-MAPK: phosphorylated MAPK; RTK: receptor tyrosine kinase

\section{Declarations}

\section{Acknowledgments}

The OVCAR3 was generous gift from Mackay Memorial Hospital Tamsui Branch.

\section{Author's contributions}

$\mathrm{KYT}$ and YJC conceived the original idea. KYT and ZYL carried out the experiments. KYT and ZYL contributed to data analyses. KYT wrote the manuscript. YJC helped in manuscript writing. YJC and SJC supervised the project. All authors read and approved the final manuscript.

\section{Funding:}

This work was supported by the Hsinchu MacKay Memorial Hospital [grant numbers MMH-HB-10513].

\section{Availability of data and materials}

The datasets used and/or analysed during the current study are available from the corresponding author on reasonable request.

\section{Ethics approval and consent to participate}

Not applicable.

\section{Consent for publication}

Not applicable. 


\section{Competing interests}

The authors declare that they have no competing interests.

\section{References}

1. Sankaranarayanan, R. and J. Ferlay, Worldwide burden of gynaecological cancer: the size of the problem. Best Pract Res Clin Obstet Gynaecol, 2006. 20(2): p. 207-25.

2. McCluggage, W.G., Morphological subtypes of ovarian carcinoma: a review with emphasis on new developments and pathogenesis. Pathology, 2011. 43(5): p. 420-32.

3. Reid, B.M., J.B. Permuth, and T.A. Sellers, Epidemiology of ovarian cancer: a review. Cancer biology \& medicine, 2017. 14(1): p. 9-32.

4. Brachova, P., et al., TP53 oncomorphic mutations predict resistance to platinum and taxanebased standard chemotherapy in patients diagnosed with advanced serous ovarian carcinoma. Int J Oncol, 2015. 46(2): p. 607-18.

5. Olivier, M., M. Hollstein, and P. Hainaut, TP53 mutations in human cancers: origins, consequences, and clinical use. Cold Spring Harbor perspectives in biology, 2010. 2(1): p. a001008-a001008.

6. Cole, A.J., et al., Assessing mutant p53 in primary high-grade serous ovarian cancer using immunohistochemistry and massively parallel sequencing. Sci Rep, 2016. 6: p. 26191.

7. Hamroun, D., et al., The UMD TP53 database and website: update and revisions. Hum Mutat, 2006. 27(1): p. 14-20.

8. Olivier, M., et al., The IARC TP53 database: new online mutation analysis and recommendations to users. Hum Mutat, 2002. 19(6): p. 607-14.

9. Oren, M. and V. Rotter, Mutant p53 gain-of-function in cancer. Cold Spring Harb Perspect Biol, 2010. 2(2): p. a001107.

10. Adorno, M., et al., A Mutant-p53/Smad complex opposes $p 63$ to empower TGFbeta-induced metastasis. Cell, 2009. 137(1): p. 87-98.

11. Muller, P.A., et al., Mutant p53 drives invasion by promoting integrin recycling. Cell, 2009. 139(7): p. 1327-41.

12. Sauer, L., et al., Mutant $p 53$ initiates a feedback loop that involves Egr-1/EGF receptor/ERK in prostate cancer cells. Oncogene, 2010. 29(18): p. 2628-37.

13. Wang, W., et al., Mutant $p 53-R 273 H$ gains new function in sustained activation of EGFR signaling via suppressing miR-27a expression. Cell Death Dis, 2013. 4: p. e574.

14. Yallowitz, A.R., et al., Mutant p53 Amplifies Epidermal Growth Factor Receptor Family Signaling to Promote Mammary Tumorigenesis. Mol Cancer Res, 2015. 13(4): p. 743-54.

15. Muller, P.A.J. and K.H. Vousden, Mutant p53 in cancer: new functions and therapeutic opportunities. Cancer cell, 2014. 25(3): p. 304-317. 
16. Hanel, W., et al., Two hot spot mutant p53 mouse models display differential gain of function in tumorigenesis. Cell Death Differ, 2013. 20(7): p. 898-909.

17. Lee, J.G., et al., Mutant $p 53$ promotes ovarian cancer cell adhesion to mesothelial cells via integrin beta4 and Akt signals. Sci Rep, 2015. 5: p. 12642.

18. Steelman, L.S., et al., Roles of the Raf/MEK/ERK and PI3K/PTEN/Akt/mTOR pathways in controlling growth and sensitivity to therapy-implications for cancer and aging. Aging, 2011. 3(3): p. 192-222.

19. Martelli, A.M., et al., The emerging multiple roles of nuclear Akt. Biochim Biophys Acta, 2012. 1823(12): p. 2168-78.

20. Mayo, L.D. and D.B. Donner, A phosphatidylinositol 3-kinase/Akt pathway promotes translocation of Mdm2 from the cytoplasm to the nucleus. Proceedings of the National Academy of Sciences of the United States of America, 2001. 98(20): p. 11598-11603.

21. Huang, W.C., et al., Nuclear translocation of epidermal growth factor receptor by Akt-dependent phosphorylation enhances breast cancer-resistant protein expression in gefitinib-resistant cells. $\mathrm{J}$ Biol Chem, 2011. 286(23): p. 20558-68.

22. Brand, T.M., et al., Nuclear EGFR as a molecular target in cancer. Radiother Oncol, 2013. 108(3): p. 370-7.

23. Vasey, P.A., Resistance to chemotherapy in advanced ovarian cancer: mechanisms and current strategies. Br J Cancer, 2003. 89 Suppl 3: p. S23-8.

24. Siwak, D.R., et al., Targeting the epidermal growth factor receptor in epithelial ovarian cancer: current knowledge and future challenges. J Oncol, 2010. 2010: p. 568938.

25. Gui, T. and K. Shen, The epidermal growth factor receptor as a therapeutic target in epithelial ovarian cancer. Cancer Epidemiol, 2012. 36(5): p. 490-6.

26. Sheng, Q. and J. Liu, The therapeutic potential of targeting the EGFR family in epithelial ovarian cancer. British journal of cancer, 2011. 104(8): p. 1241-1245.

27. Mayr, D., et al., Analysis of gene amplification and prognostic markers in ovarian cancer using comparative genomic hybridization for microarrays and immunohistochemical analysis for tissue microarrays. Am J Clin Pathol, 2006. 126(1): p. 101-9.

28. Ginath, S., et al., Expression of heparanase, Mdm2, and erbB2 in ovarian cancer. Int J Oncol, 2001. 18(6): p. 1133-44.

29. Dogan, E., et al., $p 53$ and $m d m 2$ as prognostic indicators in patients with epithelial ovarian cancer: a multivariate analysis. Gynecol Oncol, 2005. 97(1): p. 46-52.

30. Chang, S.J., et al., Proteomic investigating the cooperative lethal effect of EGFR and MDM2 inhibitors on ovarian carcinoma. Arch Biochem Biophys, 2018. 647: p. 10-32.

31. Chou, T.C. and P. Talalay, Quantitative analysis of dose-effect relationships: the combined effects of multiple drugs or enzyme inhibitors. Adv Enzyme Regul, 1984. 22: p. 27-55.

32. Hudson, L.G., et al., Activated epidermal growth factor receptor in ovarian cancer. Cancer treatment and research, 2009. 149: p. 203-226. 
33. Lafky, J.M., et al., Clinical implications of the ErbB/epidermal growth factor (EGF) receptor family and its ligands in ovarian cancer. Biochim Biophys Acta, 2008. 1785(2): p. 232-65.

34. Meier, R., et al., Mitogenic activation, phosphorylation, and nuclear translocation of protein kinase B 3 . Journal of Biological Chemistry, 1997. 272(48): p. 30491-30497.

35. Leinninger, G.M., et al., Phosphatidylinositol 3-kinase and Akt effectors mediate insulin-like growth factor-I neuroprotection in dorsal root ganglia neurons. The FASEB journal, 2004. 18(13): p. 15441546.

36. Borgatti, P., et al., Threonine 308 phosphorylated form of Akt translocates to the nucleus of PC12 cells under nerve growth factor stimulation and associates with the nuclear matrix protein nucleolin. Journal of cellular physiology, 2003. 196(1): p. 79-88.

37. Tzivion, G., M. Dobson, and G. Ramakrishnan, FoxO transcription factors; Regulation by AKT and 143-3 proteins. Biochim Biophys Acta, 2011. 1813(11): p. 1938-45.

38. Chou, T.C., Theoretical basis, experimental design, and computerized simulation of synergism and antagonism in drug combination studies. Pharmacol Rev, 2006. 58(3): p. 621-81.

39. Brosh, R. and V. Rotter, When mutants gain new powers: news from the mutant $p 53$ field. Nat Rev Cancer, 2009. 9(10): p. 701-13.

40. Arden, K.C. and W.H. Biggs, 3rd, Regulation of the FoxO family of transcription factors by phosphatidylinositol-3 kinase-activated signaling. Arch Biochem Biophys, 2002. 403(2): p. 292-8.

41. Zhang, X., et al., Akt, FoxO and regulation of apoptosis. Biochimica et Biophysica Acta (BBA) Molecular Cell Research, 2011. 1813(11): p. 1978-1986.

42. Mayo, L.D., et al., PTEN protects p53 from Mdm2 and sensitizes cancer cells to chemotherapy. Journal of Biological Chemistry, 2002. 277(7): p. 5484-5489.

43. Moll, U.M. and O. Petrenko, The MDM2-p53 interaction. Mol Cancer Res, 2003. 1(14): p. 1001-8.

44. Wang, J., et al., AKT Hyperactivation and the Potential of AKT-Targeted Therapy in Diffuse Large BCell Lymphoma. Am J Pathol, 2017. 187(8): p. 1700-1716.

45. Lo, H.W., et al., Novel prognostic value of nuclear epidermal growth factor receptor in breast cancer. Cancer Res, 2005. 65(1): p. 338-48.

46. Chaar, I., et al., Relationship between MDM2 and p53 alterations in colorectal cancer and their involvement and prognostic value in the Tunisian population. Appl Immunohistochem Mol Morphol, 2013. 21(3): p. 228-36.

47. Habashy, H.O., et al., FOXO3a nuclear localisation is associated with good prognosis in luminal-like breast cancer. Breast Cancer Res Treat, 2011. 129(1): p. 11-21.

48. Agarwal, R. and S.B. Kaye, Ovarian cancer: strategies for overcoming resistance to chemotherapy. Nature Reviews Cancer, 2003. 3: p. 502.

49. Yang, W., N.G. Dolloff, and W.S. El-Deiry, ERK and MDM2 prey on FOXO3a. Nature Cell Biology, 2008. 10: p. 125. 


\section{Figures}

A

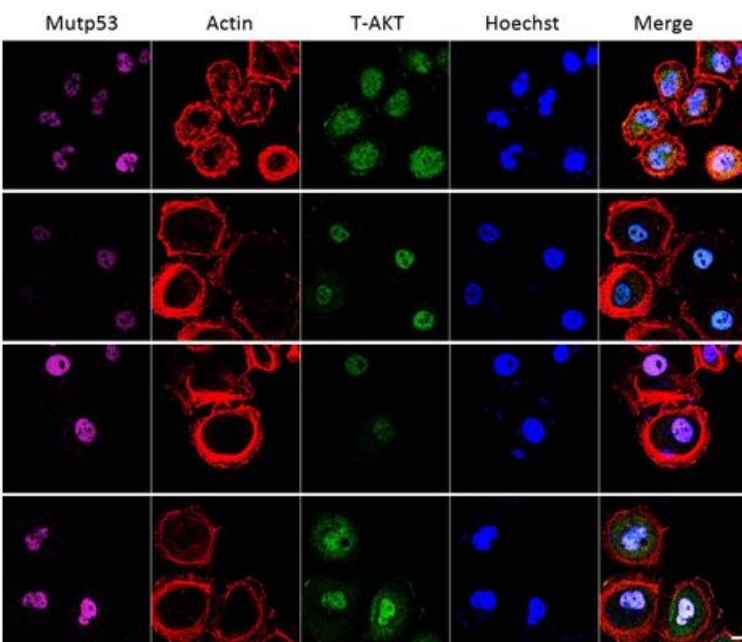

C

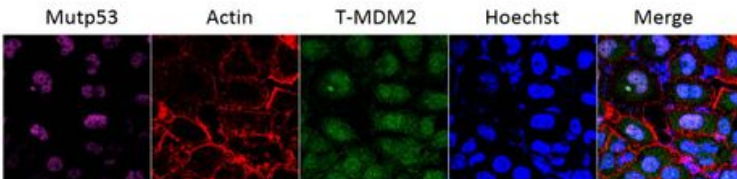

+EGF

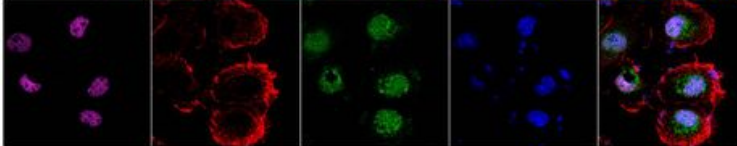

p53 R248Q overexpression

p53 R248Q overexpression +EGF

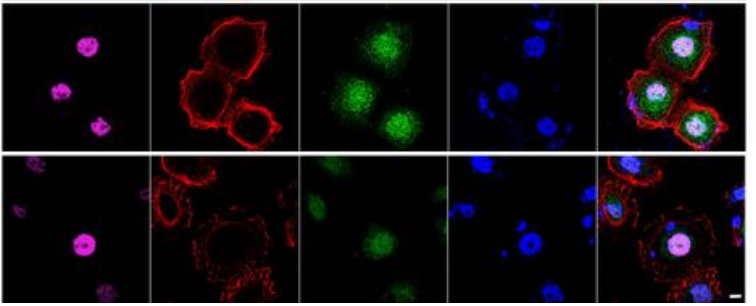

B

control

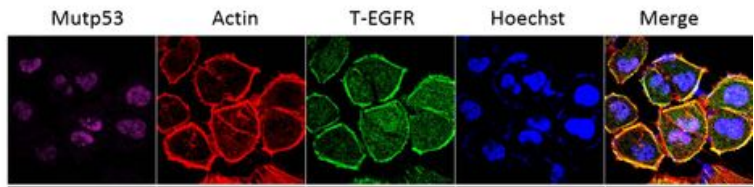

+EGF

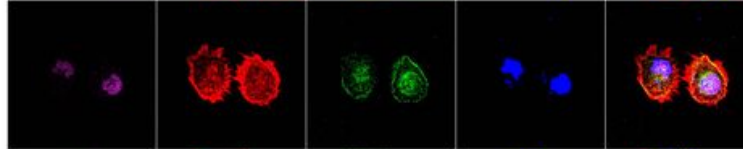

p53 R248Q

overexpression

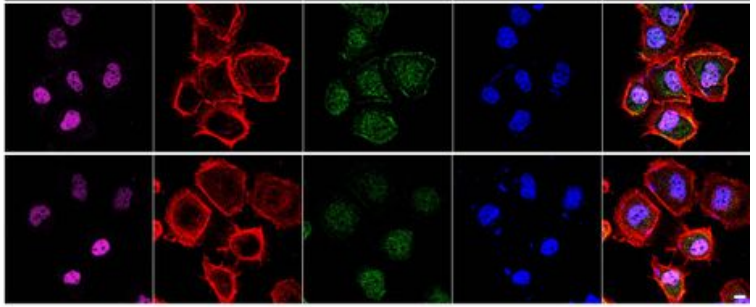

D

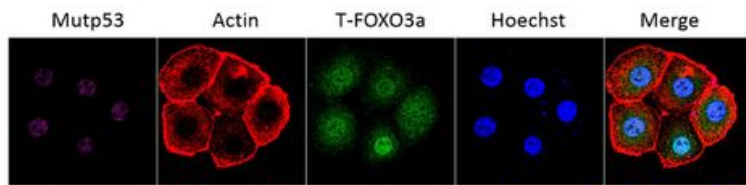

+EGF

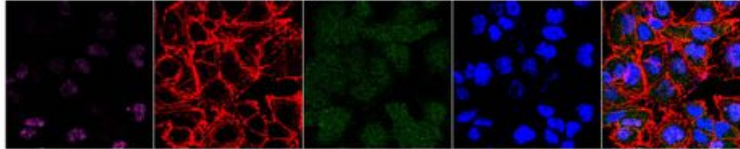

p53 R248Q overexpression

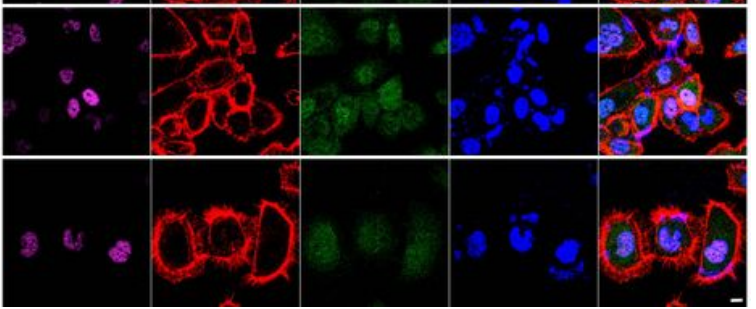

Figure 1

p53R248Q overexpression and EGF stimulation altered the intracellular localization patterns of AKT, EGFR, MDM2 and FOX03a. In this experiment, we first transfected OVCAR3 cells with or without the p53R248Q expression plasmid $(1 \mu \mathrm{g} / \mathrm{mL})$ for 24 hours and then serum-starved the cells for another 24 hours. Before confocal imaging, we stimulated the cells in the presence or absence of EGF (50 ng/mL) for 15 minutes. That is, four conditions were analyzed: the "control" group, in which the cells were serumstarved only; the "+EGF" group, in which the cells were treated with EGF after serum starvation; the "p53R248Q overexpression" group, in which the cells were transfected with p53R248Q before serum starvation; and the "p53R248Q overexpression +EGF" group, in which the cells were transfected with p53R248Q, serum-starved, and then treated with EGF. Since the cells were only transiently transfected with p53R248Q, we immunostained the cells with an anti-mutant p53 antibody to ensure that the transfected cells overexpressed p53R248Q. (A) Confocal image analysis revealed that most AKT translocates into the nucleus completely after EGF stimulation or p53R248Q overexpression. However, when the cells were overexpressed with p53R248Q and then stimulated with EGF, some AKT remained in 
the cytoplasm. (B) In the control group, most EGFR accumulated on the cell membrane, while some EGFR distributed evenly in the cytoplasm. After EGF stimulation or p53R248Q overexpression, EGFR appeared to converge and translocate toward the nucleus. When overexpressed with $\mathrm{p} 53 \mathrm{R} 248 \mathrm{Q}$ and then stimulated with EGF, EGFR could not be detected on the cell membrane. (C) In the control group, MDM2 could be detected in the cytoplasm. After EGF stimulation, MDM2 dynamically converged around the nucleus. Such phenomena could also be observed after overexpression of p53R248Q. With both p53R248Q overexpression and EGF stimulation, MDM2 translocates into the nucleus. (D) FOXO3a, which mostly accumulated in the nucleus in the control group, was evenly distributed in the cytoplasm after treatment with either EGF stimulation, p53R248Q overexpression, or both. The cells were immunostained with anti-AKT antibody, anti-EGFR antibody, anti-MDM2 antibody, anti-FOXO3a antibody (shown in green), and anti-mutant p53 antibody (shown in purple); actin in red and DNA in blue were shown by Phalloidin staining and Hoechst staining, respectively. The scale bar represents $10 \mu \mathrm{m}$.
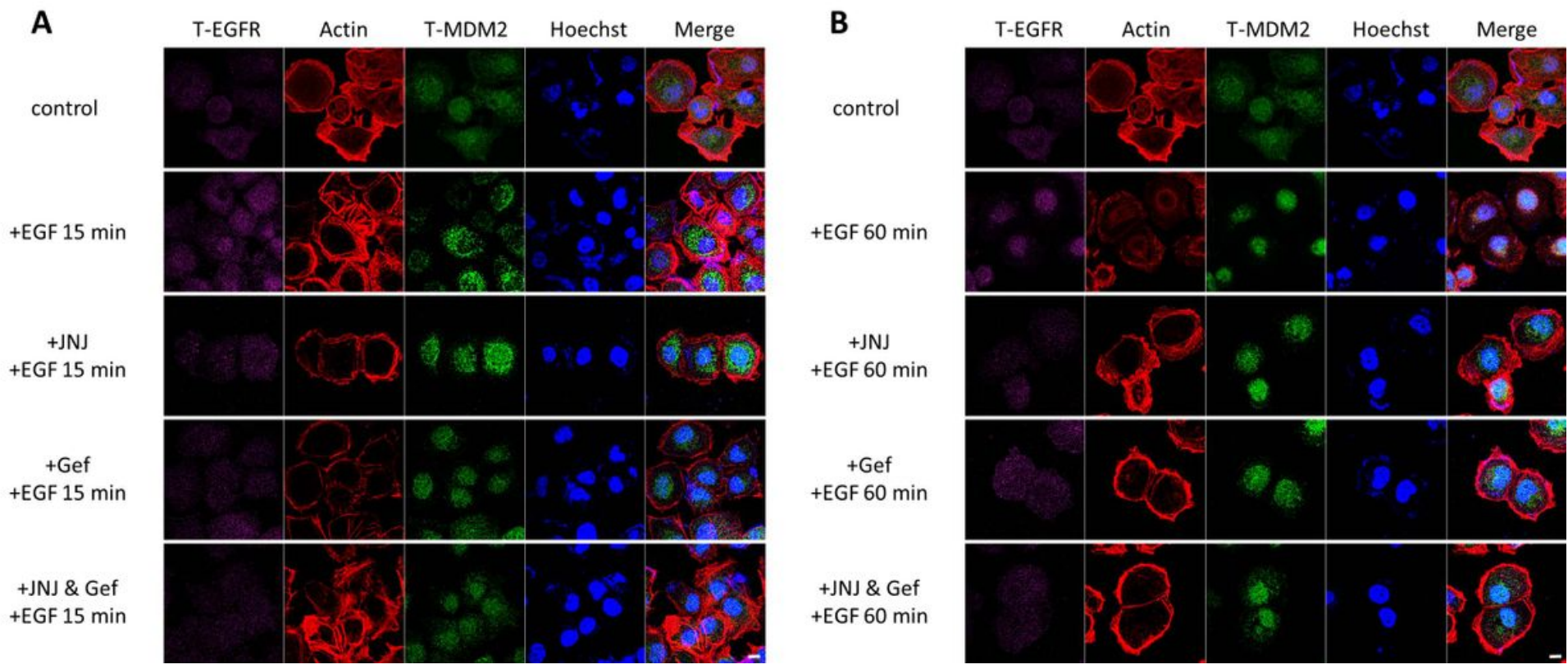

\section{Figure 2}

Cytonuclear trafficking of EGFR and MDM2 would be disrupted under combined inhibition of EGFR and MDM2 by gefitinib and JNJ. OVCAR3 cells were serum-starved while treated with or without the inhibitors under different conditions (i.e., 1.4 $\mu \mathrm{M}$ JNJ, 14.4 $\mu \mathrm{M}$ gefitinib, or $1 \mu \mathrm{M}$ JNJ + $1 \mu \mathrm{M}$ gefitinib; the IC50 concentration was derived from our previous study [30]) for 24 hours. The cells were then stimulated with EGF (50 ng/mL) for 0 (control), 15 or 60 minutes. (A) Confocal image analysis demonstrated that EGFR and MDM2, which are initially distributed in the cytoplasm, converge and translocate in sync to the periphery of the nucleus after 15 minutes of EGF stimulation. However, the convergence pattern of EGFR was disrupted after 24 hours of treatment with gefitinib, JNJ, or their combination. In comparison, the convergence pattern of MDM2 was disrupted only under the combined treatment of JNJ and gefitinib. (B) After 60 minutes of EGF stimulation, EGFR and MDM2 translocated completely into the nucleus. With combined treatment of gefitinib and JNJ for 24 hours, some MDM2 could be detected again in the cytoplasm. The cells were immunostained with anti-EGFR antibody (shown in purple) and anti-MDM2 
antibody (shown in green); actin in red and DNA in blue were shown by Phalloidin staining and Hoechst staining, respectively. The scale bar represents $10 \mu \mathrm{m}$.

A

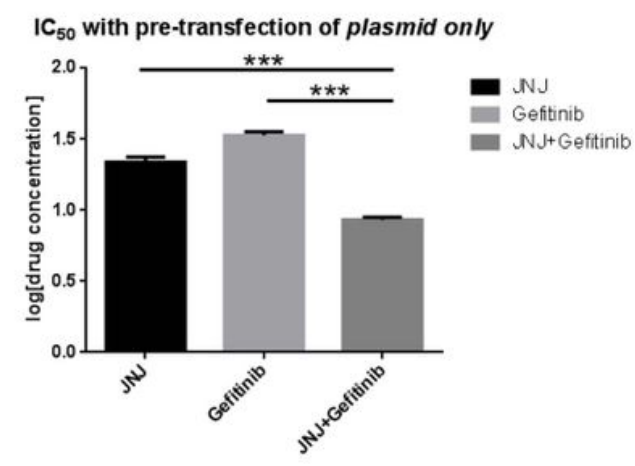

C

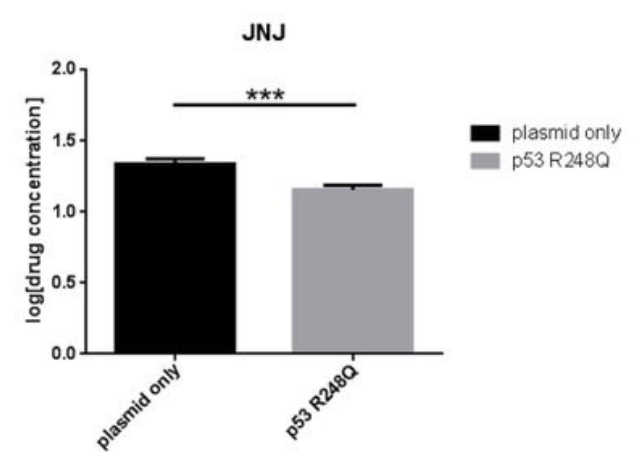

B

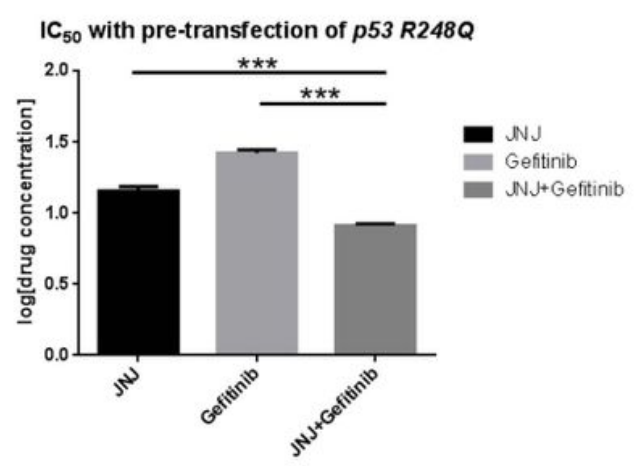

D

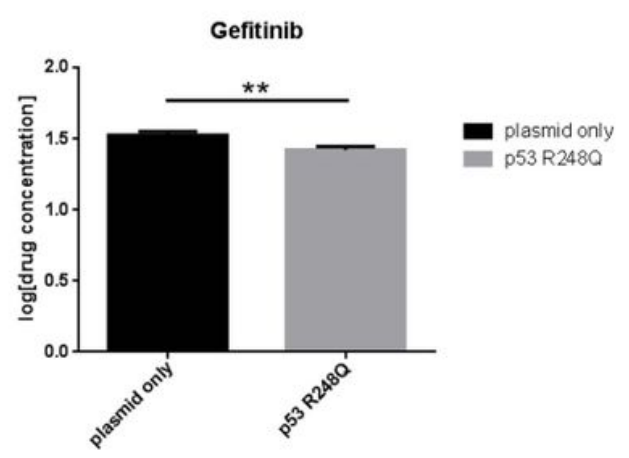

E

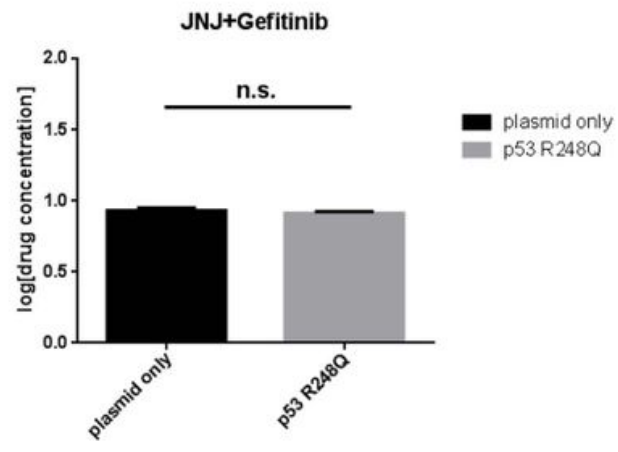

Figure 3

Overexpression of p53R248Q in OVCAR3 cells increased sensitivity to JNJ or gefitinib. In this experiment, OVCAR3 cells were pretransfected with either empty plasmid $(1 \mu \mathrm{g} / \mathrm{mL})$ or p53R248Q $(1 \mu \mathrm{g} / \mathrm{mL})$ for 18 hours and then exposed to the inhibitors alone or in combination for another 48 hours. The doses of gefitinib and JNJ ranged from 5 to $40 \mu \mathrm{M}$ and 1 to $36 \mu \mathrm{M}$, respectively. The concentrations of gefitinib and $\mathrm{JNJ}$ in combination were prepared in a 1-to-1 ratio, with doses ranging from 0.5 to $12 \mu \mathrm{M}$. The effects on cell proliferation were assessed by CCK-8 assay; the IC50 values (in log10) were calculated by GraphPad Prism. (A) In the absence or (B) in the presence of p53R248Q overexpression, the combined treatment of gefitinib and JNJ significantly reduced the IC50 values for each agent in OVCAR3 cells ( ${ }^{\star \star \star} \mathrm{p}$ $<0.001 \mathrm{vs}$. JNJ and gefitinib). (C), (D) The sensitivities to JNJ or gefitinib alone increased significantly in p53R248Q-overexpressing cells ( $* \star p<0.01 ; * \star \star p<0.001)$; (E) no significant difference was found when the combination treatment of $\mathrm{JNJ}$ and gefitinib was applied to the plasmid-only transfected cells and the p53R248Q-overexpressing cells. 
A

Plasmid only

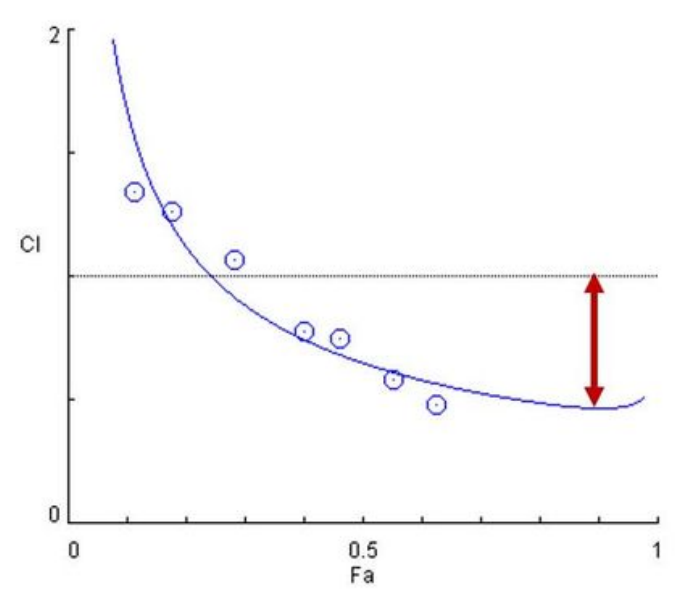

C

\section{Plasmid only}

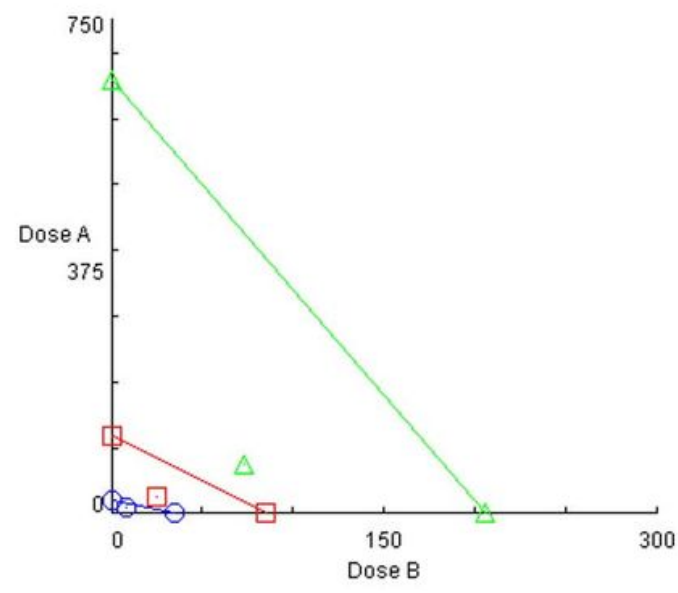

B
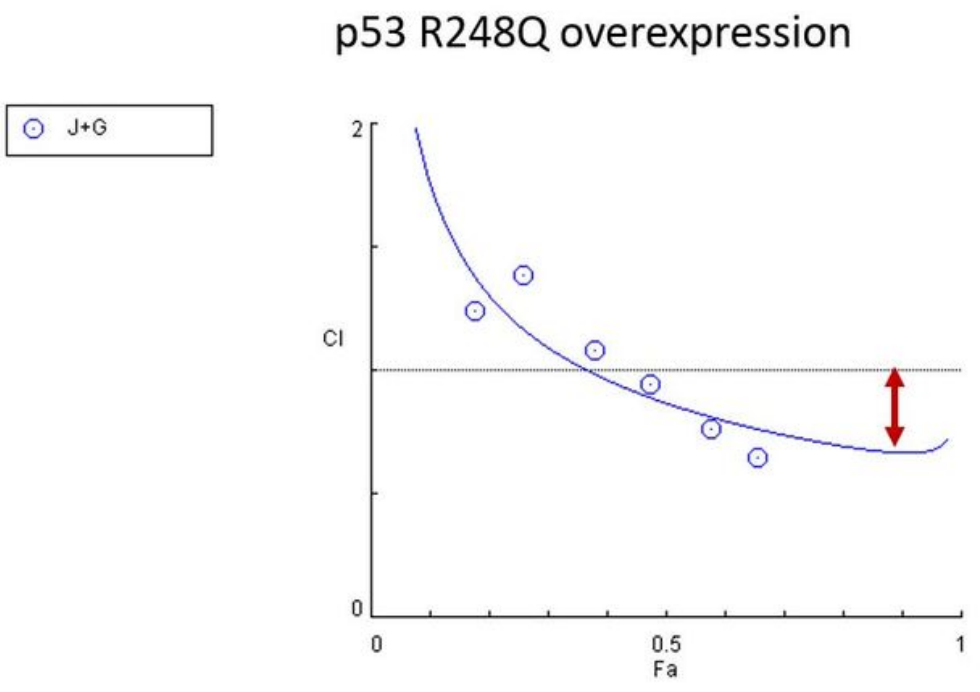

D

p53 R248Q overexpression
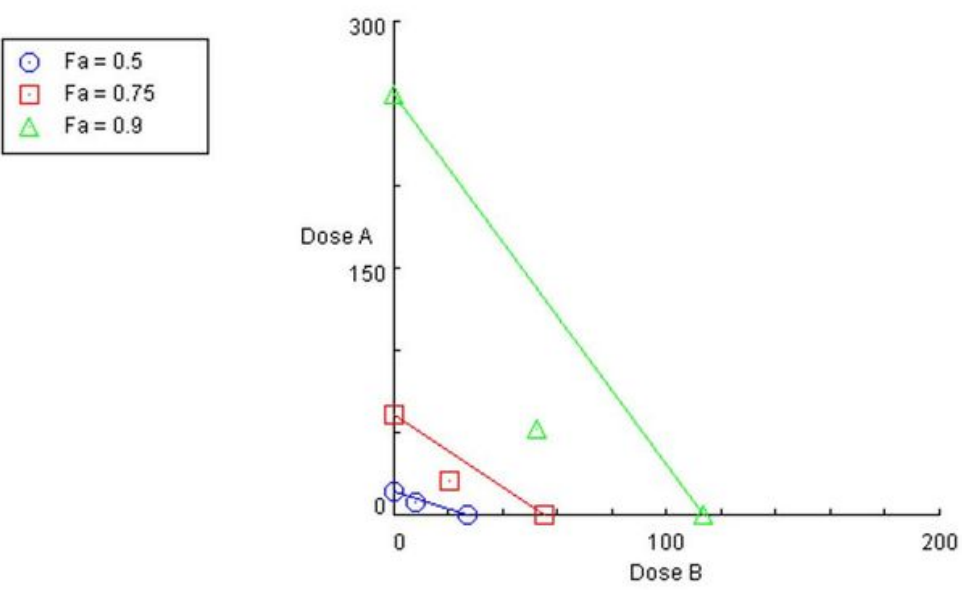

\section{Figure 4}

Synergistic effects of JNJ and gefitinib on OVCAR3 cells with different p53 statuses are shown in Fa-Cl plots and isobolograms. Based on the Chou and Talalay methods, the $\mathrm{Cl}$ values were calculated using CompuSyn software to determine the degree of synergistic effectiveness of the drug combination. The analysis revealed that overexpression of p53R248Q reduced the synergistic effect of JNJ and gefitinib. (A), (B) In the Fa-Cl plots, the fraction affected (Fa; from 0 to 1 ) represents the ratio of dead cells to total cells in response to drug treatments. Circled symbols indicate the $\mathrm{Cl}$ values of each $\mathrm{Fa}$. The $\mathrm{Cl}$ values provide a quantitative definition of synergistic $(\mathrm{Cl}<1)$, additive $(\mathrm{Cl}=1)$, and antagonistic $(\mathrm{Cl}>1)$ effects derived from the drug combination. (C), (D) In the isobolograms, the individual doses of JNJ (represented by $\mathrm{X}$-axis) and gefitinib (represented by $\mathrm{Y}$-axis) that achieve $90 \%(\mathrm{Fa}=0.9), 75 \%(\mathrm{Fa}=0.75)$, and 50\% $(\mathrm{Fa}=0.5)$ inhibitory effects are indicated by green lines, red lines, and blue lines, respectively. Symbols (ED90 is marked as green triangles; ED75 is marked as red squares; ED50 is marked as blue circles) 
above the lines, on the lines, and below the lines represent antagonistic, additive, and synergistic effects, respectively. When the symbols are located below and distant from the lines, it represents strong synergism.

A

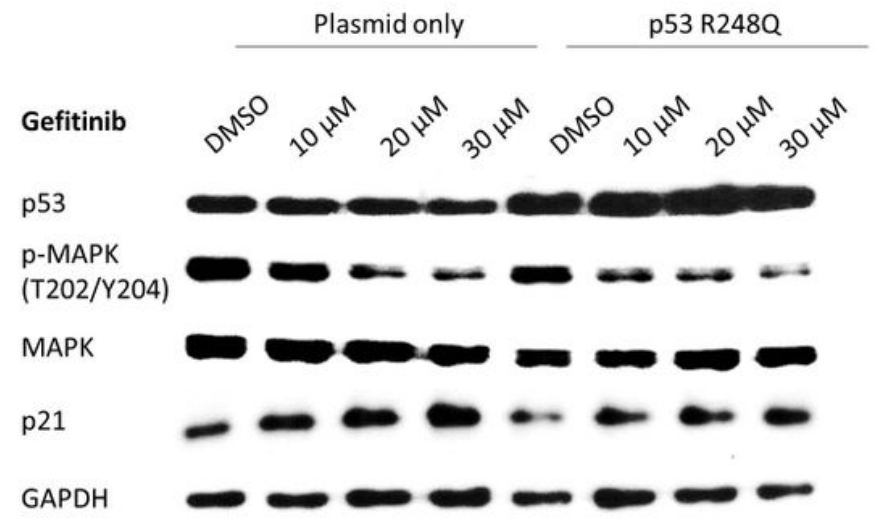

B

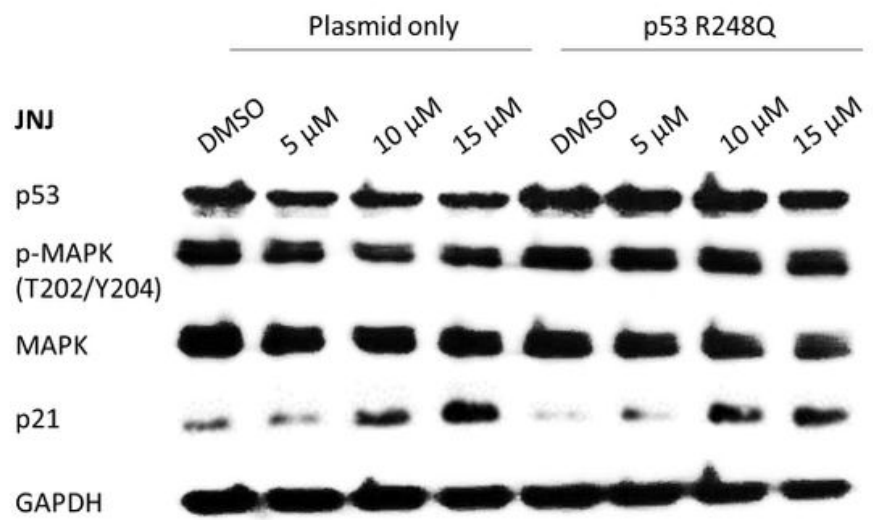

C

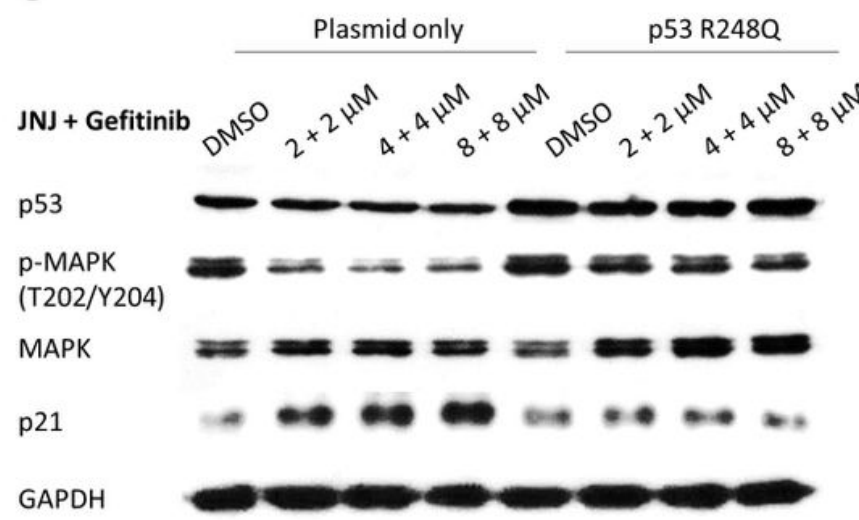

\section{Figure 5}

MAPK and p21 were involved in regulating the cell signaling responses to single or combined treatments of gefitinib and JNJ. OVCAR3 cells were pretransfected with either empty plasmid or p53R248Q plasmid before treatment with various doses of gefitinib $(10,20$ or $30 \mu \mathrm{M})$, JNJ $(5,10$, or $15 \mu \mathrm{M})$, or JNJ + gefitinib $(2+2,4+4$, or $8+8 \mu \mathrm{M})$ for 24 hours. (A) The level of $p$-MAPK was significantly reduced with increasing doses of gefitinib. The dosage-dependent inhibition of p-MAPK displayed an even more profound effect in the $\mathrm{p53R248Q}$ overexpression group. The level of p21 was upregulated in both groups with increasing doses of gefitinib. The dosage-dependent upregulation of p21 was not significantly different between the two groups. (B) With increasing doses of JNJ, the level of p-MAPK decreased slightly in the plasmid-only group, while sustained levels of p-MAPK were detected in the $\mathrm{p} 53 \mathrm{R} 248 \mathrm{Q}$ overexpression group. The level of p21 was upregulated significantly with increasing doses of JNJ. Despite the lower level of p21 in the DMSO control of the $\mathrm{p} 53 \mathrm{R} 248 \mathrm{Q}$ overexpression group, the dosage-dependent upregulation of p21 was greater in the $553248 Q$ overexpression group than in the plasmid only group. (C) With increasing doses of the JNJ-gefitinib combination, the level of p-MAPK significantly decreased in the plasmid-only group but not in the p53R248Q overexpression group. Moreover, the level of p21 increased with a profound 
effect in the plasmid-only group but not in the p53R248Q overexpression group. Phosphorylation of MAPK 1/2 (Thr202/Tyr204) was determined by immunoblot analysis using corresponding antibodies. GAPDH expression was measured to serve as a loading control.
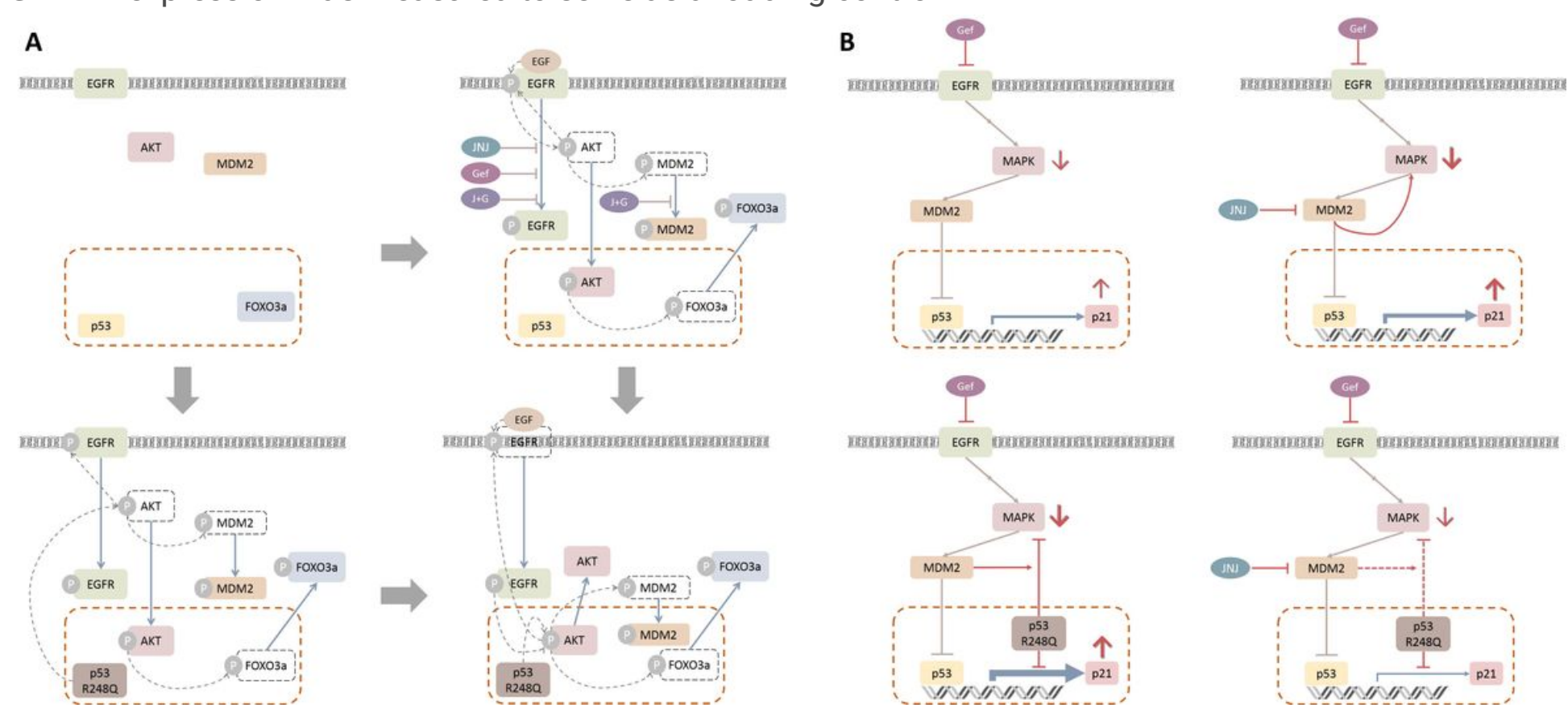

\section{Figure 6}

Hypothetical models to integrate molecular trafficking and synergistic lethal effects of gefitinib and JNJ with different p53 statuses (A) This model scheme depicts the signal transduction and molecular trafficking under p53R248Q overexpression and/or EGF stimulation in OVCAR3 cells. (B) This model scheme depicts the molecular regulation under gefitinib and/or JNJ treatments in OVCAR3 cells with different p53 statuses.

\section{Supplementary Files}

This is a list of supplementary files associated with this preprint. Click to download.

- Additionalfile1.docx 